\title{
Adaptação da Bateria de Testes EARS ao Português Europeu
}

\author{
European Portuguese EARS Test Battery Adaptation
}

Marisa ALVES ${ }^{1}$, Daniela RAMOS ${ }^{1}$, Graça OLIVEIRA ${ }^{1}$, Helena ALVES ${ }^{1}$, Ilona ANDERSON² , Isabel MAGALHÃES ${ }^{1}$, Jorge H. MARTINS ${ }^{1}$, Margarida SIMÕES ${ }^{1}$, Raquel FERREIRA ${ }^{1}$, Rita FONSECA ${ }^{1}$, Susana ANDRADE ${ }^{1}$, Luís SILVA ${ }^{1}$, Carlos RIBEIRO ${ }^{1}$, Pedro Lopes FERREIRA ${ }^{3}$

Acta Med Port 2014 Jan-Feb;27(1):23-32

\section{RESUMO}

Introdução: A utilização de instrumentos de avaliação em saúde adequados é fundamental na gestão da prestação de cuidados. A escassez, em Portugal, de instrumentos específicos para a avaliação do desempenho de crianças utilizadoras de implantes cocleares motivou o trabalho de tradução e de adaptação da bateria de testes EARS (Evaluation of Auditory Responses to Speech) para o português europeu. Esta bateria de testes é hoje um dos instrumentos mais comummente utilizados por equipas de (re)habilitação de crianças surdas com implantes cocleares em todo o mundo. O objetivo a atingir com a validação do EARS foi fornecer às equipas de (re)habilitação um instrumento que permita: (i) monitorizar a evolução individual da reabilitação; (ii) gerir um programa de (re)habilitação de acordo com resultados objetivos, comparáveis entre diferentes equipas de (re)habilitação; (iii) obter dados comparáveis com equipas internacionais; e (iv) melhorar a adesão e a motivação da família e restantes profissionais no ambulatório.

Material e Métodos: No processo de tradução e de adaptação da bateria de testes, os procedimentos adotados foram os seguintes: (i) tradução da versão inglesa para português europeu por um tradutor profissional; (ii) revisão dessa tradução realizada por um painel de especialistas constituído por otorrinolaringologistas, terapeutas da fala e técnicos de audiologia; (iii) adaptação dos estímulos de teste pela equipa de terapeutas da fala; e (iv) nova revisão por parte do painel de especialistas.

Resultados: São apresentados, para cada um dos instrumentos que compõem a bateria EARS, as adaptações introduzidas, conciliando as características e os objetivos originais dos instrumentos com as particularidades linguísticas e culturais da população portuguesa.

Discussão: São discutidas as dificuldades encontradas durante o processo de tradução e de adaptação e as soluções adotadas. São feitas comparações com outras versões da bateria EARS.

Conclusão: Considera-se que o processo de tradução e adaptação da bateria de testes EARS para o português europeu foi realizado de forma apropriada, respeitando as características dos instrumentos originais e adequando os estímulos de teste à realidade linguística e cultural da população portuguesa, cumprindo assim os objetivos propostos.

Palavras-chave: Portugal; Implantes Cocleares; Correcção de Deficiência Auditiva; Testes Auditivos; Percepção da Fala.

\section{ABSTRACT}

Introduction: The use of adequate assessment tools in health care is crucial for the management of care. The lack of specific tools in Portugal for assessing the performance of children who use cochlear implants motivated the translation and adaptation of the EARS (Evaluation of Auditory Responses to Speech) test battery into European Portuguese. This test battery is today one of the most commonly used by (re)habilitation teams of deaf children who use cochlear implants worldwide. The goal to be achieved with the validation of EARS was to provide (re)habilitation teams an instrument that enables: (i) monitoring the progress of individual (re)habilitation, (ii) managing a (re)habilitation program according to objective results, comparable between different (re)habilitation teams, (iii) obtaining data that can be compared with the results of international teams, and (iv) improving engagement and motivation of the family and other professionals from local teams.

Material and Methods: For the test battery translation and adaptation process, the adopted procedures were the following: (i) translation of the English version into European Portuguese by a professional translator, (ii) revision of the translation performed by an expert panel, including doctors, speech-language pathologists and audiologists, (iii) adaptation of the test stimuli by the team's speechlanguage pathologist, and (iv) further review by the expert panel.

Results: For each of the tests that belong to the EARS battery, the introduced adaptations and adjustments are presented, combining the characteristics and objectives of the original tests with the linguistic and cultural specificities of the Portuguese population.

Discussion: The difficulties that have been encountered during the translation and adaptation process and the adopted solutions are discussed. Comparisons are made with other versions of the EARS battery.

Conclusion: We defend that the translation and the adaptation process followed for the EARS test battery into European Portuguese was correctly conducted, respecting the characteristics of the original instruments and adapting the test stimuli to the linguistic and cultural reality of the Portuguese population, thus meeting the goals that have been set.

Keywords: Portugal; Cochlear Implants; Correction of Hearing Impairment; Hearing Tests; Speech Perception.

\footnotetext{
1. Serviço de Otorrinolaringologia. Centro Hospitalar e Universitário de Coimbra. Coimbra. Portugal.

2. MED-EL. Innsbruck. Áustria.

3. Centro de Estudos e Investigação em Saúde. Faculdade de Economia. Universidade de Coimbra. Coimbra. Portugal.

Recebido: 24 de Maio de 2013 - Aceite: 26 de Maio de 2013 | Copyright $\odot$ Ordem dos Médicos 2014
} 


\section{INTRODUÇÃO}

A utilização de instrumentos adequados de avaliação em saúde é fundamental para a gestão clínica e económica da prestação de cuidados. Em Portugal, são escassos os instrumentos específicos de avaliação para a população pediátrica com défice auditivo. A Tabela 1 apresenta uma lista dos principais instrumentos de avaliação utilizados internacionalmente e baseados no desempenho linguístico e auditivo de crianças utilizadoras de implante coclear. ${ }^{1}$

Diferentes programas de implantação coclear desenvolveram baterias de testes com esse propósito. A opção por baterias de testes e não por um teste individual deve-se à complexidade da avaliação da evolução das crianças. É o caso do Nottingham Early Assessment Package (NEAP) e do Protocolo de Avaliação de Navarra, as mais divulgadas internacionalmente. O NEAP ${ }^{2-3}$ é uma bateria de avaliação e monitorização do progresso a nível comunicativo e linguístico, desenvolvida no âmbito do programa de implantação coclear pediátrica de Nottingham, e composta pelos instrumentos listados na Tabela 2. Por outro lado, o programa de implantes cocleares do Departamento de Otorrinolaringologia da Clínica Universitária de Navarra desenvolveu um protocolo de avaliação da audição e da linguagem ${ }^{4}$ de que fazem parte os instrumentos listados na Tabela 3.

Em Portugal, a avaliação da evolução auditiva e linguística da população pediátrica com défice auditivo muitas vezes é efetuada com recurso a testes validados para crianças normo-ouvintes, às vezes mesmo sem a respetiva validação para a língua e para a cultura portuguesas. O programa de implantação coclear do Serviço de Otorrinolaringologia do Centro Hospitalar e Universitário de Coimbra ${ }^{10}$ utiliza uma bateria de avaliações da qual constam vários instrumentos e dos quais se salientam os apresentados na Tabela 4.

Face ao exposto, e encarando como insuficientes e pouco específicos os instrumentos disponíveis para a avaliação do desempenho da população pediátrica portuguesa com défice auditivo, considerou-se importante realizar a adaptação e a validação da bateria de testes EARS (Evaluation of Auditory Responses to Speech) que, sucintamente, se apresenta de seguida.

A bateria de testes EARS começou a ser desenvolvida, em 1995, por Dianne J. Allum-Mecklenburg, em cooperação com audiologistas, otorrinolaringologistas, psicólogos, psicolinguísticas e terapeutas da fala que recolheram, desenvolveram, adaptaram, combinaram e sistematizaram vários instrumentos de avaliação. Atualmente, é um dos instrumentos mais utilizados nesta área. Desde a sua apresentação, a bateria de testes EARS já foi adaptada a mais de 20 línguas, o que permite a comparação do desempenho entre crianças de diferentes línguas e culturas. ${ }^{11}$ Esta bateria de testes tem hoje definidos como objetivos: (i) avaliar o desenvolvimento da perceção auditiva em crianças com surdez severa a profunda, utilizadoras de implantes cocleares; (ii) providenciar apoio na programação e (re) habilitação destas crianças; e (iii) constituir-se como um instrumento de avaliação, a longo prazo, para crianças utilizadoras de implantes cocleares. A sua população alvo é constituída por crianças com surdez severa a profunda entre os 3 e os 10 anos de idade. ${ }^{11}$

Desde o desenvolvimento da bateria EARS que diversos estudos sobre a evolução do desempenho de crianças utilizadoras de implantes cocleares, desde a fase pré-operatória até vários anos após a implantação coclear, têm utilizado este instrumento. Alguns deles com crianças provenientes de centros de implantação coclear e mesmo de diferentes países e utilizadoras de diferentes marcas de implantes cocleares. ${ }^{11-18}$ Esta bateria de testes também foi utilizada para documentar o progresso e o benefício de crianças com défices associados. ${ }^{19}$ Os vários estudos referidos sublinham a importância da monitorização do desenvolvimento da perceção auditiva em crianças utilizadoras de implantes cocleares, bem como o seu papel no que diz respeito ao prognóstico relativamente ao desenvolvimento da linguagem e da fala. Os processos de adaptação de instrumentos de avaliação a diferentes línguas e culturas são complexos, pelo que as várias adaptações que a bateria EARS sofreu foram realizadas com extremo cuidado, para que se verificasse a possibilidade de comparação internacional dos resultados. ${ }^{20-21}$

Assim, face à insuficiência e falta de especificidade dos instrumentos de avaliação utilizados em Portugal para a avaliação do desempenho de crianças utilizadoras de implantes cocleares, revelou-se importante o trabalho de tradução e de adaptação da bateria de testes EARS ao português europeu. A escolha recaiu sobre esta bateria devido essencialmente a duas razões: a qualidade dos instrumentos que a constituem e a limitada casuística de crianças utilizadoras de implante coclear, o que cria a necessidade de testes que permitam a comparação com casos internacionais, possível utilizando uma bateria de testes já amplamente adaptada.

Tendo em conta que a utilização de instrumentos de avaliação estrangeiros sem a adequada adaptação linguística e cultural relativa aos indivíduos que se pretende avaliar pode colocar em causa a validade e a precisão da avaliação efetuada, ${ }^{22}$ considerou-se essencial a realização de um processo cuidadoso de tradução e adaptação para o português europeu e para a cultura portuguesa. Esse processo levantou dificuldades específicas, devido à necessidade de respeitar as características originais dos testes, enquadrando-os na realidade linguística e cultural portuguesa.

O presente trabalho teve então dois objetivos principais: (i) o primeiro foi a tradução e adaptação da bateria de testes EARS ao português europeu, descrita no presente artigo; (ii) o segundo foi a verificação da adequação dos estímulos verbais e visuais escolhidos para a população portuguesa, através da realização de um estudo piloto em crianças normo-ouvintes com idades compreendidas entre os 2 anos e os 4 anos e 11 meses, que será descrito num futuro artigo. Considerou-se adequado validar cultural- 
Tabela 1 - Principais instrumentos de avaliação utilizados internacionalmente com a população pediátrica que apresenta défice auditivo

\begin{tabular}{|c|c|}
\hline Instrumentos de Avaliação & Área de medição \\
\hline Meaningful Auditory Integration Scale (MAIS) ${ }^{30}$ & Capacidades auditivas e o seu uso em ambientes naturais \\
\hline Infant-Toddler Meaningful Auditory Integration Scale (IT-MAIS) ${ }^{33}$ & Capacidades auditivas e o seu uso em ambientes naturais \\
\hline Visual Habituation $(\mathrm{VH})^{34}$ & Perceção de padrões da fala \\
\hline Test of Auditory Perception of Speech for Children (TAPS) & Perceção auditiva \\
\hline Glendonald Auditory Screening Procedure (GASP) ${ }^{28}$ & Capacidade de reconhecer perguntas familiares ou do quotidiano \\
\hline Categories of Auditory Performance (CAP) ${ }^{35}$ & $\begin{array}{l}\text { Aborda o desempenho auditivo desde a resposta a sons ambientais até } \\
\text { ao desempenho com o telefone }\end{array}$ \\
\hline Auditory Speech Sound Evaluation $(\mathrm{A} \S \mathrm{E})^{36}$ & Discriminação de fonemas independentemente dos itens lexicais \\
\hline Mr. Potato Head Task ${ }^{37}$ & Reconhecimento de palavras-chave e de frases \\
\hline Escalas de Desenvolvimento da Linguagem de Reynell ${ }^{38}$ & Desenvolvimento da linguagem \\
\hline Inventário de Desenvolvimento da Comunicação de MacArthur (CDI) $)^{39}$ & Inventário das primeiras palavras utilizadas \\
\hline Rossetti Infant-Toddler Language Scales ${ }^{40}$ & Capacidades linguísticas \\
\hline Preferential Looking Paradigm (PLP) ${ }^{41}$ & Aprendizagem de associações entre sons da fala e objetos \\
\hline Maternal Speech & Estilo comunicativo dos pais \\
\hline Meaningful Use of Speech Scale (MUSS) ${ }^{31}$ & Controlo vocal, utilização da fala e estratégias comunicativas \\
\hline Babbling onset & Presença de múltiplos movimentos articulatórios \\
\hline Babbling spurt & Momento em que a frequência do balbucio aumenta significativamente \\
\hline Tait Video Analysis (TVA) ${ }^{42}$ & Desenvolvimento pré-verbal e linguístico \\
\hline Common Phrases Test ${ }^{43}$ & Compreensão de frases utilizadas no quotidiano \\
\hline Lexical Neighborhood Test (LNT) ${ }^{44}$ & Reconhecimento de palavras em formato aberto \\
\hline
\end{tabular}

Tabela 2 - Instrumentos do Nottingham Early Assessment Package

\begin{tabular}{|c|c|}
\hline Instrumentos de Avaliação & Área de medição \\
\hline Tait Video Analysis (TVA) ${ }^{42}$ & Desenvolvimento pré-verbal e linguístico \\
\hline $\begin{array}{l}\text { Pragmatics Profile of Everyday Communication Skills: Preschool } \\
\text { version (PPECS) })^{45}\end{array}$ & Capacidades sociais, de interação e conversacionais \\
\hline Stories/Narrative Assessment Procedure (SNAP) ${ }^{46}$ & Capacidades narrativas \\
\hline Profile of Actual Linguistic Skills (PALS) ${ }^{47}$ & Capacidade para usar a linguagem verbal oral \\
\hline Preschool Language Scale (PLS) ${ }^{48}$ & Desenvolvimento da linguagem a nível da compreensão e da expressão \\
\hline Listening Progress Profile $(\mathrm{LiP})^{23}$ & Monitorização de alterações no desempenho auditivo de crianças surdas \\
\hline Categories of Auditory Performance (CAP) ${ }^{35}$ & $\begin{array}{l}\text { Aborda o desempenho auditivo desde a resposta a sons ambientais até ao } \\
\text { desempenho com o telefone }\end{array}$ \\
\hline Meaningful Auditory Integration Scale (MAIS) ${ }^{49}$ & Capacidade auditiva e o seu uso nos ambientes naturais \\
\hline Profile of Actual Speech Skills (PASS) ${ }^{50}$ & Vocalizações espontâneas e padrões de fala \\
\hline Speech Intelligibility Rating (SIR) ${ }^{51}$ & Ritmo de progresso da inteligibilidade do discurso \\
\hline
\end{tabular}


Tabela 3 - Instrumentos do Programa de implantes cocleares do Departamento de Otorrinolaringologia da Clínica Universitária de Navarra

\begin{tabular}{|c|c|}
\hline Instrumentos de Avaliação & Área de medição \\
\hline Teste de identificação de vogais & Identificação de vogais; Estímulos do tipo consoante-vogal-consoante ou vogais isoladas \\
\hline Teste de identificação de consoantes & Identificação dos 13 fonemas consonânticos mais frequentes na língua castelhana \\
\hline Séries fechadas de palavras quotidianas & $\begin{array}{l}\text { Conjuntos de palavras do quotidiano em que a criança tem diante de si as listas escritas o } \\
\text { representadas graficamente }\end{array}$ \\
\hline Teste de perceção precoce da palavra & $\begin{array}{l}\text { Teste de seleção fechada com aumento progressivo da dificuldade e com o objetivo de categorizar } \\
\text { perceção da palavra }\end{array}$ \\
\hline Teste de dissílabos & Teste de eleição aberta utilizando palavras dissilábicas \\
\hline Teste de monossílabos & Teste de eleição aberta utilizando palavras monossilábicas \\
\hline Teste de frases de eleição aberta com apoio & Frases que a criança deverá repetir com o apoio de imagens relacionadas com as frases ouvidas \\
\hline
\end{tabular}

Teste de leitura labial

Categories of Auditory Performance ${ }^{35}$

Análise da voz

Registo fonológico induzido

Prova de linguagem oral de Navarra

Teste de vocabulário de imagens Peabody

Teste de Illinois de aptidões psicolinguísticas

Gael-P
Repetição de frases com acesso à leitura labial; a avaliação é feita com e sem aparelhamento

Aborda o desempenho auditivo desde a resposta a sons ambientais até ao desempenho com o telefone

Avaliação da intensidade, altura tonal, melodia e ritmo, duração, entoação e coordenação pneumofónica

Nomeação de imagens para registar as produções das crianças

Avaliação da fonologia, morfossintaxe, semântica e uso da linguagem

Avaliação do vocabulário das crianças com palavras simples, através da identificação de uma imagem entre 4 possiveis

Avaliação da compreensão auditivo-visual, associação auditivo-visual, expressão verbal-motora, integração gramático-visuo-auditiva, e memória sequencial auditiva e visuo-motora

Avaliação da linguagem a nível da compreensão e da expressão mente esta bateria em crianças normo-ouvintes com idade cronológica inferior às idades para as quais foi desenvolvida tendo em conta o conceito de idade auditiva. Em crianças com audição normal a idade auditiva equivale à idade cronológica, porém, em crianças com défice auditivo, ela corresponde ao período de tempo desde a primeira programação do processador de fala do implante coclear ou desde a primeira adaptação da prótese auditiva. Assim, a idade auditiva em crianças com défice auditivo é inferior à sua idade cronológica.

O processo de tradução e adaptação da bateria de testes EARS para o português europeu foi resultante de um protocolo entre a MED-EL Elektromedizinische Geräte $\mathrm{GmbH}$, o Centro Hospitalar de Coimbra, EPE e o Centro de Estudos e Investigação em Coimbra da Universidade de Coimbra, e foi realizado durante os anos de 2010 e 2011.

\section{MATERIAL E MÉTODOS}

\section{Bateria de testes EARS}

A bateria de testes EARS é composta pelos seguintes instrumentos de avaliação:
- $\quad$ LIP - Perfil do progresso auditivo ${ }^{23}$

O LIP (Listening Progress Profile) é um teste que permite avaliar a perceção auditiva de vários sons, incluindo a fala, assim como o desenvolvimento das capacidades auditivas em crianças com implantes cocleares. Neste teste são observadas as seguintes competências e/ou comportamentos: reação a sons ambientais; identificação de sons ambientais; resposta ao toque de tambor (estimulada); resposta (estimulada) a outros instrumentos musicais; resposta à voz (estimulada e espontânea); discriminação de toque de tambor alto face a baixo e um toque face a toques repetidos; reação aos sons /a, i, u, m, s, J/; discriminação de sons da fala entre altos e baixos, um som da fala face a repetidos sons da fala, sons de fala longos e breves, três dos seis sons de Ling e os seis sons de Ling; discriminação de dois nomes de familiares com diferente número de sílabas; identificação do próprio nome em silêncio. A pontuação para cada item é de 0,1 ou 2, em que 0 corresponde a 'nunca/não sabe', 1 corresponde a 'às vezes' e 2 corresponde a 'sempre'. 
Tabela 4 - Instrumentos utilizados no âmbito do Protocolo de Avaliação Pós-Implantação Coclear da Unidade de Implantes Cocleares do Serviço de Otorrinolaringologia do Centro Hospitalar e Universitário de Coimbra

Instrumentos de Avaliação Área de medição

Teste de monossílabos

Teste de números

Teste de repetição de frases sem apoio

Teste de repetição de frases sem apoio ao telefone

Teste de 25 palavras

Teste de 25 palavras ao telefone

Teste de 100 palavras

Teste de 100 palavras ao telefone

Teste de compreensão de estruturas complexas ${ }^{52}$

Teste de Avaliação da Linguagem na Criança (TALC) ${ }^{53}$

Grelha de Avaliação da Linguagem - Nível Escolar $(\mathrm{GOL}-\mathrm{E})^{54}$

Teste de articulação Verbal ${ }^{55}$

Speech Intelligibility Rating ${ }^{51}$

Grelha de avaliação das características vocais (GACV)

Categories of Auditory Performance ${ }^{35}$

Sons de Ling

Meaningful Auditory Integration Scale (MAIS) ${ }^{49}$

Teste de identificação de consoantes

Inventário de Desenvolvimento da Comunicação de MacArthur (CDI) ${ }^{39}$

Meaningful Use of Speech Scale (MUSS) ${ }^{31}$

Teste de identificação de intervalo no ruído

Teste de padrão de duração

Teste de padrão de frequência

Teste de fala filtrada

Teste de fala no ruído

Teste de escuta dicótica - Dissílabos alternados e sobrepostos
Teste de eleição aberta utilizando palavras monossilábicas

Teste de eleição aberta utilizando números

Teste de eleição aberta composto por frases que devem ser repetidas sem qualquer tipo de apoio

Semelhante ao anterior, mas aplicado através do telefone

Teste de eleição aberta utilizando palavras do quotidiano, que devem ser repetidas

Semelhante ao anterior, mas aplicado através do telefone

Teste de eleição aberta utilizando palavras do quotidiano, que devem ser repetidas

Semelhante ao anterior, mas aplicado através do telefone

Estruturas sintáticas complexas

Semântica, morfossintaxe e pragmática

Semântica, morfossintaxe e fonologia

Produção dos fonemas consonânticos do português

Ritmo de progresso da inteligibilidade do discurso

Intensidade, altura tonal, ressonância nasal, entoação e coordenação pneumofonoarticulatória

Aborda o desempenho auditivo desde a resposta a sons ambientais até ao desempenho com o telefone

Identificação dos sons de Ling

Capacidade auditiva e o seu uso nos ambientes naturais

Identificação dos fonemas consonânticos da língua portuguesa

Inventário das primeiras palavras utilizadas

Controlo vocal, utilização da fala e estratégias comunicativas

Processamento auditivo - processamento temporal

Processamento auditivo - processamento temporal

Processamento auditivo - processamento temporal

Processamento auditivo - teste monoaural de baixa redundância

Processamento auditivo - teste monoaural de baixa redundância

Processamento auditivo - teste dicótico 
- $M D P$ - Monossílabos-Dissílabos-Polissílabos ${ }^{24}$

O MDP MTP (Monosyllabic-Trochee-Polysyllabic) é um teste que avalia a capacidade para identificar diferentes padrões silábicos. Tem três versões, respetivamente, com 3, 6 e 12 palavras. Tem como objetivos determinar a capacidade da criança para identificar diferentes padrões silábicos variando entre uma sílaba, duas sílabas e mais de duas sílabas. É solicitado à criança que aponte ou repita as palavras, com apoio nas imagens ou nos objetos apresentados previamente pelo avaliador. O teste é aplicado sem a ajuda da leitura labial (estimulação auditiva somente). Cada palavra é pronunciada uma vez e é pedido à criança que responda. As respostas são pontuadas a nível do padrão silábico corretamente identificado e da palavra corretamente identificada.

- Palavras monossilábicas em contexto fechado 25

O Teste de Palavras Monossilábicas em Contexto Fechado (Monosyllable Closed-Set Test) é um teste que avalia a capacidade para identificar palavras monossilábicas familiares. É composto por duas listas, de 4 e 12 palavras respetivamente. É solicitado à criança que aponte ou repita as palavras, com apoio nas imagens ou nos objetos apresentados previamente pelo avaliador. $O$ teste é aplicado sem a ajuda da leitura labial (estimulação auditiva somente). Cada palavra é pronunciada uma vez e é solicitado à criança que responda, sendo atribuído um ponto por cada resposta correta.

- Teste de frases em contexto fechado ${ }^{26}$

O Teste de Frases em Contexto Fechado (Closed-set sentence test) tem como objetivo demonstrar a capacidade para identificar palavras familiares co-articuladas. É constituído por matrizes com imagens: o nível A tem quatro matrizes $2 \times 3$ (a criança apenas deve considerar dois itens); o nível B1 tem duas matrizes de $3 \times 3$ (a criança deve escolher de entre três itens); o nível B2 tem duas matrizes de $3 \times 4$ (a criança deve escolher de entre três itens); e o nível $C$ tem duas matrizes de $4 \times 4$ (a criança deve escolher de entre quatro itens). $\mathrm{O}$ avaliador coloca a matriz à frente da criança e solicita-Ihe que aponte para as palavras que ouviu ou que repita a frase. O teste deverá ser aplicado sem a ajuda da leitura labial (estimulação auditiva somente). Cada frase é dita uma vez e é solicitado à criança que responda. Cada palavra correta é pontuada com (+) e cada palavra incorreta com (-). O resultado final para cada matriz é o número total de palavras corretas.

- Palavras monossilábicas em contexto aberto ${ }^{27}$

O Teste de Palavras Monossilábicas em Contexto Aberto (Monosyllable Open-Set Test) avalia a capacidade para reconhecer palavras monossilábicas. É composto por duas listas de dez palavras, sendo aplicado sem apoio da leitura labial (estimulação auditiva somente). Cada palavra é dita uma vez e pede-se à criança que responda. As respostas são pontuadas a nível do fonema correto e da palavra correta.

- GASP - Procedimento de rastreio auditivo de Glendonald ${ }^{28}$

O GASP (Glendonald Auditory Screening Procedure) é um teste que avalia a capacidade de reconhecimento de perguntas simples. É composto por 10 questões e deverá ser aplicado sem a ajuda da leitura labial (estimulação auditiva somente). Cada frase é dita uma vez e é solicitado à criança que responda. Pontua-se com 1 ponto se a resposta estiver correta e com 0 se estiver incorreta.

- COT - Teste de objetos comuns ${ }^{29}$

O teste COT (Common Objects Token Test) procura avaliar a capacidade de compreensão de frases, através do cumprimento de ordens. São utilizados os seguintes materiais: 4 carros, 4 helicópteros, 4 aviões e 4 barcos nas cores vermelho, azul, verde, amarelo, 1 comboio e 4 círculos, também um de cada cor vermelho, azul, verde, amarelo. Depois de se verificar que a criança conhece todos os objetos e todas as cores, é solicitado à criança que oiça cada uma das ordens e que depois desempenhe a tarefa solicitada. A pontuação diz respeito a toda a frase, sendo a resposta da criança classificada como correta ou incorreta; pontua-se com 1 ponto se a resposta estiver correta e com 0 se estiver incorreta.

- MAIS - Escala de Integração Auditiva ${ }^{30}$

A escala MAIS (Meaningful Auditory Integration Scale) foi concebida para avaliar as reações espontâneas da criança ao som, no seu ambiente diário. É constituída por 10 perguntas e a informação para cada uma delas é obtida através de questões indiretas que o próprio entrevistador vai adaptando às características de personalidade, de relacionamento e culturais do entrevistado. A pontuação para cada pergunta tem uma amplitude de 0 (o mais baixo) a 4 (o mais alto) pontos. A pontuação final surge em termos do número total de pontos nos 40 pontos possíveis.

- MUSS - Escala de Utilização da Fala ${ }^{31}$

A escala MUSS (Meaningful Use of Speech Scale) foi concebida para avaliar o uso da fala pela criança, em situações do dia-a-dia. É constituída por 10 perguntas e a informação relativa a cada uma delas é recolhida através da técnica de entrevista aberta. A pontuação para cada pergunta tem uma amplitude de 0 (o mais baixo) a 4 (o mais alto) pontos e a pontuação final surge através do número total de pontos nos 40 pontos possíveis.

\section{Procedimentos}

No processo de tradução e de adaptação da bateria de testes, os procedimentos adotados foram os seguintes: (i) realização de uma tradução da versão inglesa para português europeu por um tradutor profissional; (ii) revisão dessa tradução realizada por profissionais especialistas nas áreas de otorrinolaringologia, terapia da fala e audiologia; (iii) adaptação, pela equipa de terapeutas da fala, dos estímulos utilizados nos testes, procurando respeitar as características originais de cada teste, tendo em conta as particularidades linguísticas e culturais da população a estudar; e (iv) realização de nova revisão por parte de toda a equipa de profissionais.

Atendendo a que se trata de um instrumento de avaliação já validado em várias línguas, durante o processo de tradução e adaptação da bateria EARS para o português 
europeu foi consultada a versão espanhola da mesma bateria, tendo em conta as proximidades culturais e linguísticas entre Portugal e Espanha.

De entre as dificuldades que surgiram durante este processo, uma das mais relevantes foi o facto de que a tradução para o português europeu de muitos dos estímulos utilizados em alguns dos testes (e.g., palavras, sons, onomatopeias) alteravam as características do teste original, ou porque o termo resultante da tradução tinha pouca utilização na nossa língua (e, portanto, o resultado do teste seria influenciado pelo desconhecimento desse significado) ou, ainda, pela alteração do número de sílabas, nos testes em que esse é um aspeto essencial do instrumento de avaliação. Assim, houve necessidade de, para vários dos estímulos utilizados em alguns dos testes, selecionar novos estímulos que não só respeitassem as características originais do instrumento, mas também respeitassem as particularidades linguísticas e culturais da população portuguesa.

Em alguns dos testes desta bateria, parte dos estímulos necessitam de ser passíveis de serem representados por objetos ou miniaturas. Este facto constituiu-se como outra dificuldade no processo, devido à impossibilidade de aceder a objetos ou miniaturas que representassem alguns dos estímulos inicialmente selecionados, pelo que foi necessária a revisão dessa seleção. Noutras situações, foram necessários somente pequenos ajustes, por exemplo, na ordem de apresentação das imagens no teste de frases em contexto fechado (Tyler-Holstad), para que houvesse adequação à estrutura sintática das frases em português europeu.

Relativamente aos instrumentos MAIS e MUSS, uma adaptação para o português europeu já tinha sido realizada por Ferreira e Silva. ${ }^{21}$ Assim, essa adaptação foi adotada para este trabalho, tendo sido introduzidas as alterações necessárias, nomeadamente devido à introdução de uma versão direcionada para professores.

\section{RESULTADOS}

A tradução, que envolveu cerca de 300 mil carateres, foi realizada por um tradutor profissional, após o que foi revista para garantir o rigor dos termos clínicos utilizados. Para além da tradução, houve, no entanto, necessidade de proceder a alterações que respeitassem não só as características originais dos instrumentos mas, principalmente, as particularidades linguísticas e culturais da população portuguesa. Apresenta-se, de seguida, para cada um dos instrumentos que compõem a bateria EARS, as adaptações efetuadas.

\section{- LIP - Perfil do progresso auditivo}

$\mathrm{Na}$ tarefa de discriminação entre um som da fala e repetidos sons da fala substituiu-se o hop, hop hop hop (versão inglesa) por ão, ão ão ão, uma vez que se considerou que o canguru, que surge nas imagens da versão inglesa, não constitui uma referência forte na cultura portuguesa, pelo que se considerou mais adequado utilizar o cão.

$\mathrm{Na}$ tarefa de discriminação entre sons da fala longos e breves, foram substituídas os sons maaa, maaaaaaaa (representados por imagens de ovelhas, na versão inglesa) para muu, muuuuuuuu (representados por imagens de vacas), uma vez que o som onomatopaico para a ovelha, em português europeu, não corresponde ao som onomatopaico para o mesmo animal em inglês.

$\mathrm{Na}$ tarefa de discriminação de três dos seis sons de Ling introduziram-se duas pranchas (LIP-12 A e LIP-12 B) que permitem trabalhar a distinção entre os seis sons de Ling. $\mathrm{Na}$ versão inglesa existe uma prancha para discriminação dos cinco sons de Ling (com imagens representativas dos sons /a, i, u, s, J/); na versão portuguesa a prancha permite a discriminação dos seis sons de Ling (com imagens representativas dos sons /a, i, u, m, s, J/). As tarefas de discriminação de dois nomes de familiares com diferente número de sílabas e a identificação do próprio nome mantiveram-se na versão portuguesa.

\section{- MDP - Monossílabos-Dissílabos-Polissílabos}

$\mathrm{Na}$ versão com três palavras, em inglês, são apresentadas as palavras fish (monossílabo), baby (dissílabo) e elephant (polissílabo). A tradução simples dos estímulos de teste alteraria as características originais do teste, pelo que se procuraram outras palavras. Uma vez que, nesta versão de três palavras, os estímulos podem ser apresentados através de objetos, foi necessário escolher palavras passíveis de ser representadas dessa forma, procurando-se, simultaneamente, que fizessem parte do vocabulário comummente dominado por crianças pequenas. Optou-se pelas palavras pá (monossílabo), bebé (dissílabo) e cavalo (polissílabo), mantendo-se, assim, somente uma das palavras da versão inglesa.

No teste MDP de seis palavras, a versão inglesa, para além das três palavras do teste anterior, apresenta as palavras cow (monossílabo), monkey (dissílabo) e banana (polissílabo). Para este teste já não há a necessidade de os estímulos poderem ser apresentados através de objetos, mas somente através de imagens. Para além das palavras pá, bebé e cavalo, que foram escolhidas para a versão de três palavras, foram acrescentadas as palavras três (monossílabo), carro (dissílabo) e sapato (polissílabo).

No teste MDP de doze palavras, a versão inglesa, para além das seis palavras do teste anterior, inclui as palavras ball e tree (monossílabos), pencil e flower (dissílabos), butterfly e telephone (polissílabos). Para além das palavras pá, três, bebé, carro, cavalo e sapato, que foram escolhidas para a versão de seis palavras, foram selecionadas céu e mar (monossílabos), bola e lápis (dissílabos) e girafa e banana (polissílabos).

\section{- Palavras monossilábicas em contexto fechado}

No teste de quatro palavras, a versão inglesa apresenta as palavras spoon, ball, tree e cow. A tradução simples dos estímulos deste teste alteraria as características originais do teste, pelo que se procurou encontrar outras palavras monossilábicas que pertencessem ao vocabulário das crianças da faixa etária que se pretende avaliar e que 
pudessem ser representadas através de objetos. Face a estas necessidades, optou-se pelas palavras flor, cão, pau e rei.

No teste de doze palavras, a versão inglesa apresenta, para além das quatro palavras anteriormente referidas, as palavras horse, book, shoe, doll, sun, cat, rock e ship. Para este teste, para além das palavras flor, cão, pau e rei, escolhidas para a versão de quatro palavras, foram ainda selecionadas as palavras mão, sol, luz, pé, mãe, pai, ler e dói.

- Teste de frases em contexto fechado (Tyler-Holstad)

$\mathrm{Na}$ matriz nível B1 (matriz 5) na versão portuguesa, procedeu-se à alteração da palavra counts (versão inglesa) por leva.

Na matriz nível B2 (matriz 7) na versão portuguesa, a palavra cowboy (versão inglesa) foi alterada pela palavra cantora e a palavra carries (versão inglesa) pela palavra conta (versão portuguesa).

Na matriz nível C (matriz 10), a palavra cowboy (versão inglesa) foi alterada pela palavra cantora (versão portuguesa).

Cada alteração a nível das palavras utilizadas foi acompanhada da alteração da imagem correspondente. Foram ainda necessários ajustes na ordem de apresentação das imagens, em todas as matrizes, para que houvesse adequação à estrutura sintática das frases em português europeu.

- Palavras monossilábicas em contexto aberto

Na primeira lista do teste de palavras monossilábicas em contexto aberto, a versão inglesa apresenta as palavras boat, house, bean, nut, man, lip, ring, leg, match e soap. A tradução simples destes estímulos alteraria também as características originais do teste, pelo que se procurou encontrar outras palavras monossilábicas que pertencessem ao vocabulário comum de crianças pequenas portuguesas, tendo-se optado pelas palavras pão, ver, sal, dor, mil, bom, sim, deu, sai e eu.

$\mathrm{Na}$ segunda lista do teste de palavras monossilábicas em contexto aberto, a versão inglesa apresenta as palavras goat, mouse, seal, bus, boot, ship, song, lake, watch e nose. Na versão portuguesa as palavras mel, não, tu, som, cor, Rui, fim, noz, pôr e chão foram selecionadas, de acordo com os objetivos do teste.

- GASP - Procedimento de rastreio auditivo de Glendonald

Neste teste, foi feita a tradução das perguntas, não tendo havido necessidade de introduzir quaisquer alterações adicionais.

- COT - Teste de objetos comuns

Neste teste, foi feita a tradução das ordens, não tendo também havido necessidade de introduzir quaisquer alterações adicionais.
- MAIS - Escala de Integração Auditiva

Relativamente a este instrumento, uma adaptação para o português europeu já tinha sido realizada ${ }^{32}$ Assim, essa adaptação foi adotada para o presente trabalho, tendo apenas sido necessária a introdução das perguntas $1 c$ e $2 b$ e de uma versão direcionada para professores, que não faziam parte da versão originalmente adaptada.

\section{- MUSS - Escala de Utilização da Fala}

Também neste caso, uma adaptação para o português europeu já tinha sido realizada. ${ }^{32}$ Assim, essa adaptação foi adotada para o presente trabalho. No entanto, foi também necessária a introdução de uma versão direcionada para professores.

\section{DISCUSSÃO}

A tradução e a adaptação cultural e linguística de instrumentos de avaliação em saúde apresentam vários desafios.

Como já foi referido anteriormente, atendendo a que se trata de um instrumento de avaliação já validado em várias línguas, durante o processo de tradução e adaptação da bateria EARS para o português europeu foi consultada a versão espanhola da mesma bateria, tendo em conta as proximidades culturais e linguísticas entre Portugal e Espanha.

Considera-se relevante realçar que, para os testes de palavras monossilábicas em contexto fechado e palavras monossilábicas em contexto aberto, os autores da versão espanhola optaram por utilizar palavras dissilábicas. De facto, a língua inglesa apresenta uma maior frequência de palavras monossilábicas que comummente fazem parte do vocabulário de crianças pequenas e que, mais facilmente, são representadas por objetos ou imagens do que nas línguas portuguesa e espanhola. Porém, e apesar das dificuldades sentidas na escolha vocabular de monossílabos para a versão portuguesa, considerou-se importante a manutenção destes dois testes com palavras monossilábicas, uma vez que assim se cumpre um dos objetivos da realização deste trabalho, que diz respeito à possibilidade de comparação de resultados com crianças utilizadoras de implantes cocleares falantes de línguas diferentes, respeitando as características originais dos instrumentos. Esta situação poderá revestir a versão portuguesa de uma complexidade superior às das versões inglesa, pela maior frequência de palavras monossilábicas no vocabulário de crianças pequenas, e espanhola, pela substituição por dissílabos que, contudo, pode ser compensada por um reforço de treino daqueles vocábulos específicos.

As dificuldades relativas às discrepâncias de frequência de palavras e de características acústicas dos sons entre as línguas portuguesa e inglesa foram colmatadas pela equipa de validação, com base na experiência e na aplicação prática de opções alternativas que o trabalho diário com crianças utilizadoras de implantes cocleares permite.

Para que este trabalho pudesse ser considerado concluído, foi essencial a realização de um estudo piloto em 
crianças com audição normal com idades compreendidas entre os dois anos e os quatro anos e 11 meses. Considerou-se importante validar culturalmente esta bateria em crianças com audição normal com idade cronológica inferior às idades para as quais foi desenvolvida, tendo em conta o conceito de idade auditiva, já referido anteriormente. Esse estudo-piloto já foi realizado e será descrito num futuro artigo.

\section{CONCLUSÃO}

Considera-se que o processo de tradução e adaptação da bateria de testes EARS para o português europeu, apesar da sua complexidade, foi realizado de forma apropriada, respeitando as características dos instrumentos originais e adequando os estímulos de teste à realidade linguística e cultural da população portuguesa.

As equipas de (re)habilitação de crianças surdas utilizadoras de implantes cocleares passam a ter um instrumento de trabalho que lhes permite avaliar a evolução da criança,

\section{REFERÊNCIAS}

1. Vlastarakos PV, Proikas K, Papacharalampous G, Exadaktylou I, Mochloulis G, Nikolopoulos TP. Cochlear implantation under the first year of age - The outcomes. A critical systematic review and meta-analysis. Int J Pediatr Otorhinolaryngol. 2010;74:119-26.

2. Dyar D, Nikolopoulos TP. Monitoring progress: the role and remit of a speech and language therapist. In: Barry McCormick SA, ed. Cochlear Implants for Young Children: The Nottingham Approach to Assessment and Rehabilitation. Londres: Whurr Publishers; 2003.

3. Nikolopoulos TP, Archbold SM, Gregory S. Young deaf children with hearing aids or cochlear implants: early assessment package for monitoring progress. Int J Pediatr Otorhinolaryngol. 2005;69:175-86.

4. Huarte A, Molina M, Manrique M, Olleta I, García-Tapia R. Protocolo para la valoracion de la audicion y el lenguaje, en lengua española, en un programa de implantes cocleares. Acta Otorrinolaringol Esp. 1996;47:S1-14.

5. Chora JR, Matos TD, Martins JH, Alves MC, Andrade SM, Silva LF, et al. DFNB1-associated deafness in Portuguese cochlear implant users: prevalence and impact on oral outcome. Int J Pediatr Otorhinolaryngol. 2010;74:1135-9.

6. Martins JH, Alves M, Andrade S, Januário J, Ramos D, Ferreira M, et al. $(\mathrm{Re})$ habilitação auditiva através de Implante Coclear: Avaliação audiológica, da linguagem, fala e voz (estudo preliminar). Rev Port Otorrinol Cir Cérvico-Facial. 2008;46:231-5.

7. Martins JH, Januário J, Alves M, Ramos D, Alves H, Silva L, et al. Estudo comparativo de discriminação de listas de palavras e frases efectuadas em voz viva e através de telefone em pacientes com implante coclear. Rev Port Otorrinolaringol Cir Cérvico-Facial. 2010;48:181-9.

8. Peixoto C, Alves M, Martins JH, Cortesão R, Bastos J, Ribeiro C. Implante coclear - estarão as suas complicações associadas a alterações da percepção da fala? Cad Otorrinolaringol. 2012, 30 out:1-6.

9. Alves M, Ramos D, Alves H, Martins JH, Silva L, Ribeiro C. Desempenho auditivo e perceção de consoantes em adultos utilizadores de implante coclear. Cad Otorrinolaringol. 2012;4:1-4.

10. Andrade $S$, Peixoto $C$, Alves $M$, et al. Reabilitação auditiva por implante Coclear na população geriátrica. Cad Otorrinolaringol. 2012;18:1-6.

11. Esser-Leyding B, Anderson I. EARS ${ }^{\circledast}$ (Evaluation of Auditory Responses to Speech): An internationally validated assessment tool for children provided with cochlear implants. ORL J Otorhinolaryngol Relat Spec. 2012;74:42-51.

12. Allum JH, Greisiger R, Straubhaar S, Carpenter MG. Auditory perception and speech identification in children with cochlear implants tested with the EARS protocol. Br J Audiol. 2000;34:293-303.

13. Szuchnik J, Skarzyński H, Geremek A, Zawadzki R. Results of total deafness treatment in young pre- and postlingually deafened children. Scand Audiol 2001;30:S42-4.

14. Baumgartner WD, Pok SM, Egelierler B, Franz P, Gstoettner W, Hamzavi J. The role of age in pediatric cochlear implantation. Int $\mathrm{J}$ monitorizar os procedimentos efetuados, apresentar dados comparáveis com resultados de equipas estrangeiras e fornecer dados objetivos. Simultaneamente, permite um acompanhamento sistemático aos pais, assim como aos decisores políticos e potencia a existência de decisões de gestão adequadas em programas de saúde.

Como continuação deste projeto surge a verificação da adequação dos estímulos verbais e visuais escolhidos para a população infantil portuguesa, através da realização de um estudo piloto em crianças com audição normal, que será descrito num futuro artigo.

\section{CONFLITOS DE INTERESSE}

Os autores não declararam quaisquer conflitos de interesse.

\section{FONTES DE FINANCIAMENTO}

Os autores não mencionam quaisquer fontes de financiamento.

Pediatr Otorhinolaryngol. 2002;62:223-8.

15. Sainz M, Skarzynski H, Allum JH, Helms J, Rivas A, Martin J, et al. Assessment of auditory skills in 140 cochlear implant children using the EARS protocol. ORL J Otorhinolaryngol Relat Spec. 2003;65:91-6.

16. Anderson I, Weichbold V, D'Haese PS, Szuchnik J, Quevedo MS, Martin $\mathrm{J}$, et al. Cochlear implantation in children under the age of two - what do the outcomes show us? Int J Pediatr Otorhinolaryngol. 2004;68:425-31.

17. Anderson I, Weichbold V, D'Haese P. Three-year follow-up of children with open-set speech recognition who use the MED-EL cochlear implant system. Cochlear Implants Int. 2004;5:45-57.

18. Anderson I, Pitterl M, Skarzynski H, Evans R, Godey B, Sainz MQ, et al. Long-term data on children implanted with a short electrode array. Int J Pediatr Otorhinolaryngol. 2005;69:157-64.

19. Hamzavi J, Baumgartner WD, Egelierler B, Franz P, Schenk B, Gstoettner W. Follow up of cochlear implanted handicapped children. Int J Pediatr Otorhinolaryngol. 2000;56:169-74.

20. Weichbold V, Anderson I, D'Haese P. Validation of three adaptations of the Meaningful Auditory Integration Scale (MAIS) to German, English and Polish. Int J Audiol. 2004;43:156-61

21. Tsiakpini L, Esser B, D'Haese PS. Greek adaptation of EARS test battery. Logoped Phoniatrics Vocol. 2004;29:51-5.

22. Giusti E, Befi-Lopes DM. Tradução e adaptação transcultural de instrumentos estrangeiros para o Português Brasileiro. Pró-Fono Rev Atual Cient. 2008;20:207-10.

23. Archbold S. Monitoring process in children at the preverbal stage. In: B McCormick, S Archbold, S Sheppard Eds. Cochlear Implants for Young Children. London: Whurr; 1994. p.197-213.

24. Erber NP, Alencewicz CM. Audiologic evaluation of deaf children. JSHD. 1976;41:256-67.

25. Schneider A, Leyrer M, Pilkington MC, Allum D. Monosyllable ClosedSet Test. Innsbruck: MED-EL; 1996.

26. Tyler RS, Holstad BA. A closed-set speech perception test for hearingimpaired children. lowa City: Dep Otolaryngol; 1976.

27. Schneider A, Leyrer M, Pilkington MC, Allum D. Monosyllable Open-Set Test. Innsbruck: MED-EL; 1995.

28. Erber NP. Auditory Training. Washington: Alexander Graham Bell Association for the Deaf; 1982.

29. Anderson I, Martin J, Costa A, Jamieson L, Bailey E, Plant G, et al. Validation of the Common Objects Token (COT) Test for children with cochlear implants. Deafness Educ Int. 2005;7:154-70.

30. Robbins AM, Renshaw JJ, Berry SW. Evaluating meaningful auditory integration in profoundly hearing-impaired children. Am J Otol. 1991;12:S114-50.

31. Robbins AM, Osberger MJ. Meaningful use of speech scale. Indianapolis: Indiana University School of Medicine; 1992.

32. Ferreira PL, Silva LF. Avaliação da eficácia da reabilitação áudio-oral em crianças com surdez profunda. Rev Port Otorrinolaringol Cirurgia 
Cérvico-Facial. 2005;43:219-39.

33. Robbins AM, Koch DB, Osberger MJ, Zimmerman-Phillips S, KishonRabin L. Effect of age at implantation on auditory-kkill development in infants and toddlers. Apresentado no $9^{\circ}$ Symposium on Cochlear Implants in Children. Washington; 2003.

34. Houston DM, Pisoni DB, Kirk KI, Ying EA, Miyamoto RT. Speech perception skills of deaf infants following cochlear implantation: a first report. Int J Pediatr Otorhinolaryngol. 2003;67:479-95

35. Nikolopoulos TP, Archbold SM, O'Donoghue GM. The development of auditory perception in children following cochlear implantation. Int $\mathrm{J}$ Pediatr Otorhinolaryngol. 1999;49:S189-91.

36. Govaerts PJ, Daemers K, Yperman M, De Beukelaer C, De Saegher G, De Ceulaer G. Auditory speech sounds evaluation (A§E): a new test to assess detection, discrimination and identification in hearing impairment. Cochlear Implants Int. 2006;7:97-106.

37. Robbins AM. The Mr. Potato Head Task. Indianapolis: Indiana University School of Medicine; 1994.

38. Edwards S, Fletcher P, Garman M, Hughes A, Letts C, Sinka I. The Reynell Developmental Language Scales III. The University of Reading Edition. Windsor: NFER-Nelson; 1997.

39. Camaioni L, Caselli MC, Longobardi E, Volterra V. A parent report instrument for early language assessment. First Language. 1991;11:345-59.

40. Rossetti LM. Infant-toddler assessment: An interdisciplinary approach. Chicago: APA; 1990.

41. Hehar SS, Nikolopoulos TP, Gibbin KP, O'Donoghues GM. Surgery and functional outcomes in deaf children cochlear implants before age 2 years. Arch Otolaryngol Head Neck Surg. 2002;128:11-4

42. Tait M. Video analysis: a method of assessing changes in preverbal and early linguistic communication following cochlear implantation. Ear Hear. 1993;14:378-89.
43. Robbins AM, Renshaw JJ, Osberger MJ. Common phrases test Indianapolis: Indiana University School of Medicine; 1990.

44. Kirk KI, Pisoni DB, Osberger MJ. Lexical effects on spoken word recognition by pediatric cochlear implant users. Ear Hear. 1995;16:470-81.

45. Dewart $\mathrm{H}$, Summers $\mathrm{S}$. The pragmatics profile of early communication skills. Windsor: NFER-NELSON; 1988.

46. Starczewski $\mathrm{H}$, Lloyd $\mathrm{H}$. Using the stories/narrative assessment procedure (SNAP) to monitor language and communication changes after a cochlear implant: a case study. Deafg Educ Int. 1999:1:137-54.

47. Allen S, Dyar D. Profiling linguistic outcomes in young children after cochlear implantation. Am J Otol. 19978;18:s127-8.

48. Zimmerman IL, Steiner VG, Pond RE. PLS-3: Preschool language scale-3. San Antonio: The Psychological Corporation; 1992.

49. McConkey RA, Renshaw JJ, Bury SW. Meaningful Auditory Integration Scale. Evaluating meaningful auditory integration in profoundly deaf children. Am J Otol. 1991;12:S144-50.

50. Dyar D, Nikoloulos TP. Speech and language outcomes. In: B McCormick, S Archbold (Eds.), Cochlear Implants for Young Children London: Whurr; 2003. p. 327-82.

51. Allen MC, Nikolopoulos TP, O'Donoghue GM. Speech intelligibilityin chilcredf after cochlear implantation. Am J Otol. 1998;19:742-6.

52. Sim-Sim I. Avaliação da linguagem oral: Um contributo para o conhecimento do desenvolvimento linguístico das crianças portuguesas. Lisboa: Fundação Calouste Gulbenkian; 2001.

53. Kay ES, Tavares MD. Teste de Avaliação da Linguagem na Criança: TALC. Lisboa: Oficina Didáctica; 2007.

54. Sua-Kay E, Santos ME. Grelha de Avaliação da Linguagem - nível escolar (GOL-E). Alcoitão: Escola Superior de Saúde do Alcoitão; 2003.

55. Guimarães I, Grilo M. Teste de articulação verbal. Lisboa: Fisiopraxis 2004. 
Marisa ALVES, Daniela RAMOS, Graça OLIVEIRA, Helena ALVES, Ilona ANDERSON, Isabel MAGALHÃES, Jorge H. MARTINS, Margarida SIMÕES, Raquel FERREIRA, Rita FONSECA, Susana ANDRADE, Luís SILVA, Carlos RIBEIRO, Pedro Lopes FERREIRA

\section{Adaptação da Bateria de Testes EARS ao Português Europeu Acta Med Port 2014:27:23-32}

Publicado pela Acta Médica Portuguesa, a Revista Científica da Ordem dos Médicos

Av. Almirante Gago Coutinho, 151

1749-084 Lisboa, Portugal.

Tel: +351218428215

E-mail: submissao@actamedicaportuguesa.com

www.actamedicaportuguesa.com

ISSN:0870-399X | e-ISSN: 1646-0758

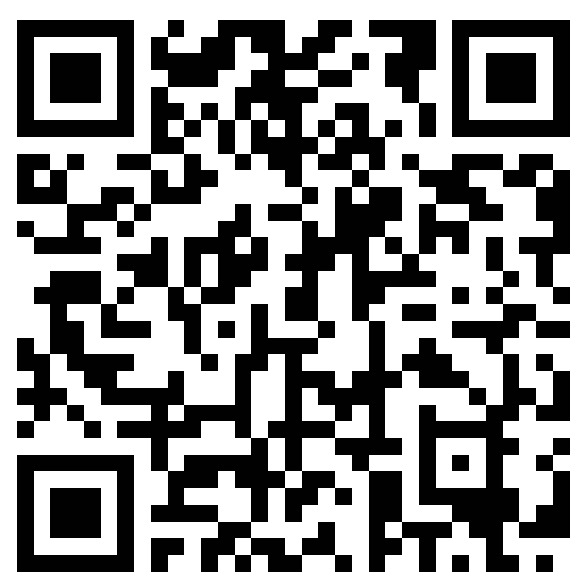

